\section{Cytochrome $c$ Oxidase Partial \\ Deficiency-Associated Leigh Disease Presenting as an Extrapyramidal Syndrome}

\section{ABSTRACT}

Leigh disease is a subacute neurodegenerative disorder characterized by symmetric necrotic lesions in the basal ganglia, cerebellum, thalamus, brain stem, and optical nerves and caused by altered oxidative phosphorylation. We describe the clinical, biochemical, neuroimaging, and molecular studies of a 19-year-old boy with early-onset Leigh disease manifesting as severe extrapyranidal disorder with generalized dystonia and choreoathetosis. He was born of healthy parents after an uneventful pregnancy and delivery. At the age of $2 \frac{1}{2}$ years, after a minor respiratory infection, he developed unstable, broad-based gait and tremor of the hands. These symptoms persisted for the next several years, when ataxia became more prominent. Difficulty in swallowing, dysarthria, trunk dystonia, and marked dyskinesia of the arms and hands gradually developed. Nystagmus, transient ptosis, and strabismus also appeared. Abnormal laboratory findings included elevated plasma and cerebrospinal fluid lactate and pyruvate, with an abnormal lactate/pyruvate ratio. Cranial computed tomography and magnetic resonance imaging demonstrated signs of cerebellar atrophy, bilateral and symmetric hypodensities in the lentiform nucleus and thalamus, and transient hyperintensities of cerebral peduncles in $\mathrm{T}_{2}$-weighted sequences suggestive of Leigh disease. Muscle biopsy revealed isolated fiber atrophy, necrotic fibers undergoing phagocytosis, and no ragged-red fibers. The measured catalytic activity of cytochrome $c$ oxidase in skeletal muscle homogenates demonstrated a partial cytochrome $c$ oxidase deficiency. No abnormalities in the mitochondrial genome and in the $S U R F-1$ gene were found. The boy is currently receiving levodopa therapy, creatine monohydrate, and a high dosage of thiamine and lipoic acid, his condition is stabilized, and extrapyramidal symptoms are less pronounced. ( $J$ Child Neurol 2001;16:616-619).

Leigh disease, or subacute necrotizing encephalomyelopathy, is a progressive neurodegenerative disorder characterized by multiple symmetric necrosis (spongy degeneration) with gliosis, demyelination, and capillary proliferation in the brain stem, basal ganglia, thalamus, cerebellum, and optical nerves. ${ }^{1.2}$ These pathologic changes are found in association with a number of different biochemical and genetic defects of mitochondrial energy generation, suggesting a common and limited response in the brain to this form of metabolic insult. ${ }^{3}$ Defined causes include the X-linked El $\alpha$ subunit of the pyruvate dehydrogenase complex, ${ }^{4}$ respiratory chain complex I, ${ }^{5}$ cytochrome $c$ oxidase, ${ }^{6-9}$ and a biotinidase deficiency. ${ }^{10}$ Leigh disease has also been found in association with point mutations in the mitochondrial DNA-encoded adenosine triphosphatase 6 subunit, ${ }^{11}$ point mutations in mitochondrial transfer ribonucleic acid genes, ${ }^{12}$ and a mutation in the $\mathrm{F}_{\mathrm{p}}$ subunit of the succinate dehydrogenase complex. ${ }^{13}$ More recently, point mutations in the SURF1 gene have been discovered in patients with isolated cytochrome $c$ oxidase deficiency. ${ }^{14}$ The defined biochemical defects account fol approximately $50 \%$ of all patients, and it is clear that there mus be many more biochemical abnormalities than hitherto to detect which lead to this characteristic pattern of neuropathology. ${ }^{15}$

The clinical presentation and course of the disease vary con. siderably as the clinical picture reflects both the variability of the lesions and their tendency to predominate in the basal ganglia brain stem, and cerebellum. Leigh disease usually affects infants anc children but can occur at any age. The diagnosis can be confirmed only by postmortem examination and is based on well-characterized neuropathologic criteria. ${ }^{2}$ Recently, clinical and radiologic correlates have emerged that permit a diagnosis of Leigh disease with a high degree of probability before death. Elevated blood and cere brospinal fluid lactate values are now a recognized metabolic marker for Leigh disease and signify a disturbance in mitochondrial function. Common clinical manifestations include psychomotor retardation, usually with regression, and signs of brainstem dysfunction including respiratory abnormalities, nystagmus and ophthalmoplegia, cerebellar dysfunction, and pyramidal and extrapyramidal signs.' Movement disorders can be prominent, presumably resulting from the frequent involvement of the basal ganglia. However, only approximately $25 \%$ of all patients have signs of movement disorders, with dystonia being the most common. ${ }^{1-1,1 t}$

We present here the clinical, neuroimaging, biochenical, and molecular studies of a 19-year-old boy with early-onset Leigh disease manifesting as severe extrapyramidal disorder with generalized dystonia and choreoathetosis owing to a partial cytochrome $c$ oxidase deficiency.

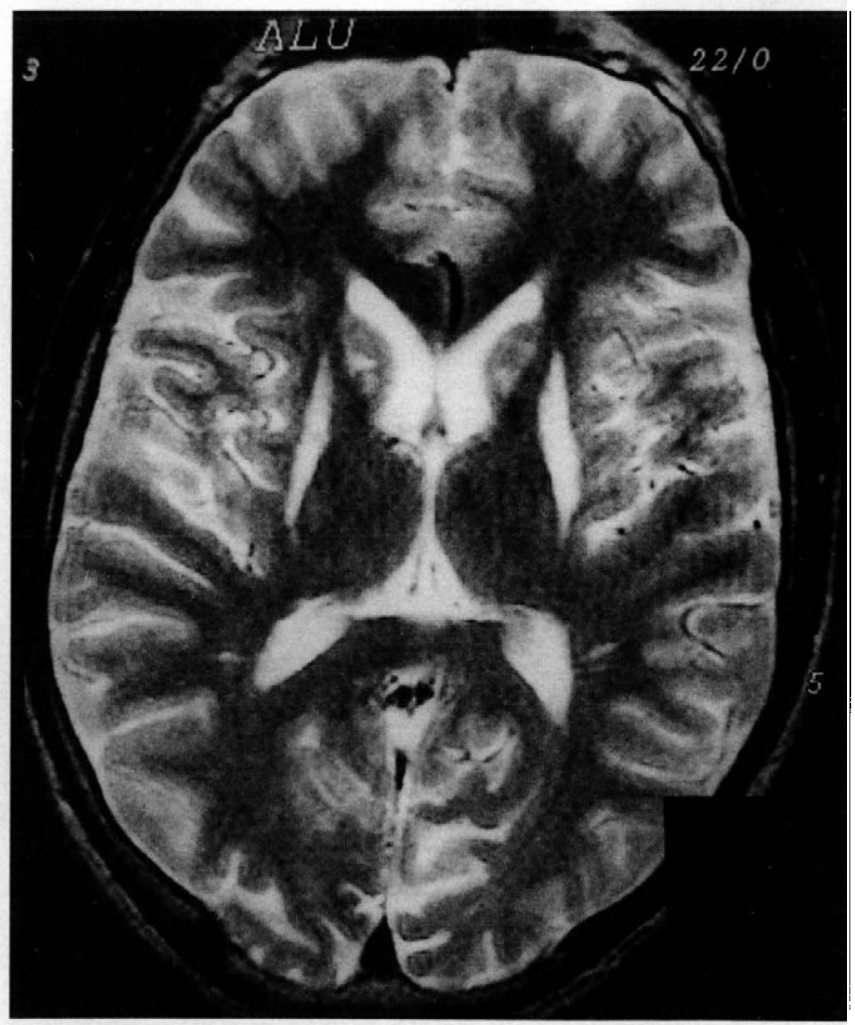

Figure 1. $\mathrm{MRIT}_{2}$-weighted axial image shows hyperintensity of the lentiform and caudate nuclei bilaterally. 


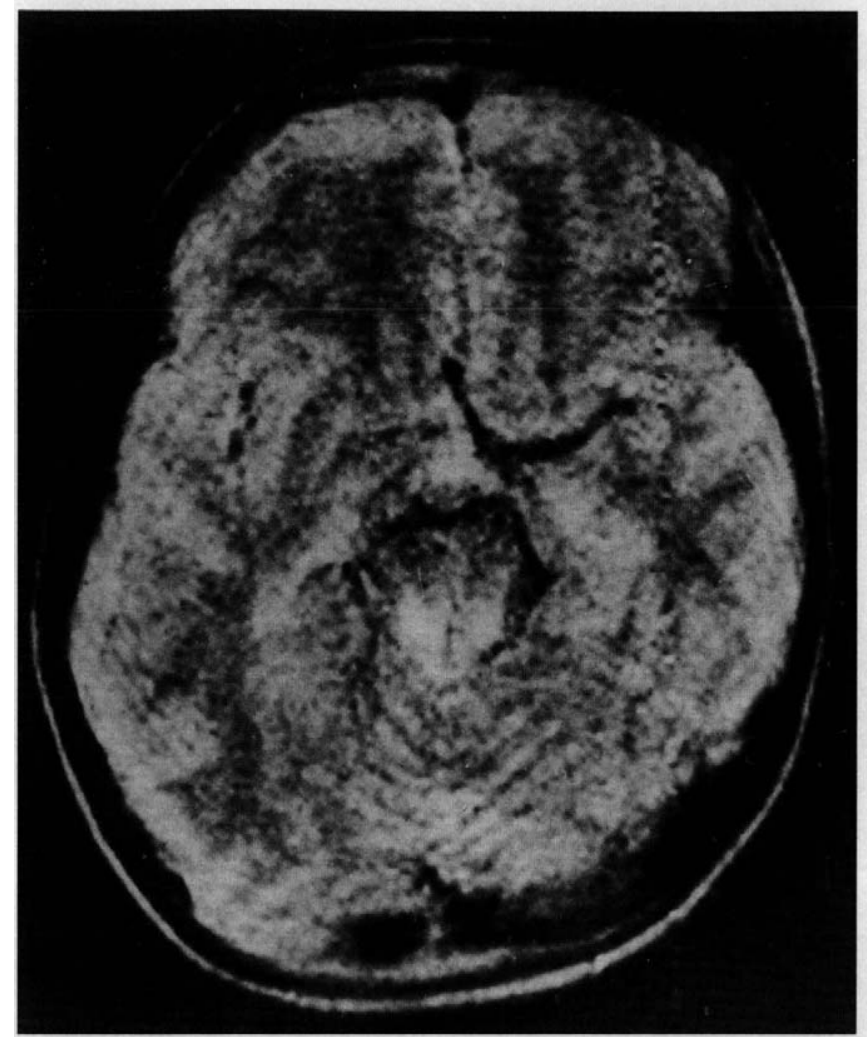

Figure 2. $\mathrm{MRIT}_{2}$-weighted axial image revealed an abnormal signal in the region of the cerebral peduncles.

\section{Case Report}

Our patient is the first-born son of healthy, nonconsanguineous parents with no family history of neurologic disease. Pregnancy, delivery, and early developmental milestones were normal. At the age of $2 \frac{1}{2}$ years, on the 10th day of respiratory infection, he developed febrile diarrhea and was hospitalized owing to dehydration. The next day, unstable ataxic gait and tremor were noticed. The cerebrospinal fluid findings were normal. The computed tomography (CT) brain scan performed 1 month later revealed marked cerebellar atrophy. Thereafter, he continued to be more ataxic and gradually developed swallowing and articulation difficulties. His speech became slurred at the age of 7 years, and, at that time, dystonic posturing of the limbs appeared with marked dyskinesia of the arms and hands. Two years later, he developed horizontal and vertical nystagmus, transient right eyelid ptosis, and divergent strabismus. The dystonia increased progressively to become generalized, involving the upper and lower extremities, trunk, and orofacial musculature. Intellectual functions have been preserved. Subsequent CT scanning at the age of $8 \frac{1 / 2}{2}$ years revealed cerebellar atrophy as previously observed, with additional symmetric bilateral radiolucencies involving the globus pallidus and caudate nucleus. First magnetic resonance imaging (MRI) scan of the brain performed at the age of $81 / 2$ years showed $\mathrm{T}_{\mathrm{N}}$-weighted images with high signal intensity abnormalities of the lentiform nucleus (Figure 1), with hyperintense areas in the thalamus and cerebral

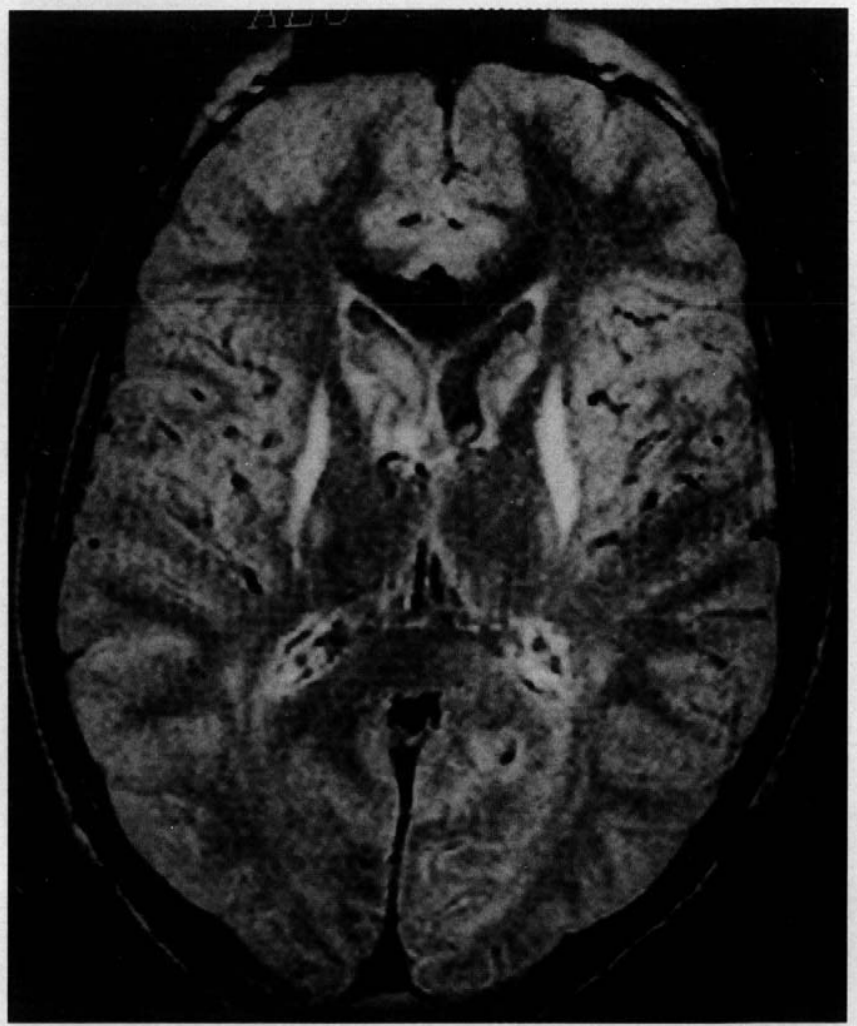

Figure 3. MRI fluid-attenuated inversion-recovery sequences at the age of 19 years demonstrated abnormal signal density in the structures of the basal ganglia.

peduncles (Figure 2). Between the ages of 7 and 19 years, the clinical picture has remained fairly stable, with dysphagia, dysarthria, gait ataxia, dystonia, and choreoathetosis.

The patient was first referred to us at the age of 15 years. At that time, neurologic examination displayed normal cognitive functions, severe dysarthria, dysphagia, gait ataxia, and dystonic quadriplegia of fluctuating postural tone with limb choreoathetosis. Signs of pyramidal dysfunction included brisk tendon reflexes with bilateral ankle clonus. Babinski reflexes were positive. There was no evidence of sensory dysfunction.

The following laboratory studies were unremarkable: complete blood count, serum electrolytes, glucose, ammonia, liver and renal function tests, serum copper and ceruloplasmin, 24-hour urinary copper excretion, carnitine in serum and urine, lysosomal enzymes, very-long-chain fatty acids, serum and urinary amino acids, urinary organic acids, oligosaccharides, and mucopolysaccharides. Mild lactic acidosis was present, with serum levels of fasting lactate varying from 2.00 to $2.98 \mathrm{mmol} / \mathrm{L}$ (normal $<2.44 \mathrm{mmol} / \mathrm{L}$ ), pyruvate levels in the normal range, and normal 24-hour urinary lactate excretion. Serum lactate levels showed an intermittent mild increase after oral glucose loading up to $2.41 \mathrm{mmol} / \mathrm{L}$ with pyruvate levels of $0.045 \mathrm{mmol} / \mathrm{L}$ (normal $<0.09 \mathrm{mmol} / \mathrm{L}$ ), giving a lactate/pyruvate ratio of 54 . A lumbar puncture was performed revealing slightly elevated levels of lactate to 2.75

Table 1. Biochemical Analysis of the Respiratory Chain Enzymes Activities in Muscle Tissue

\begin{tabular}{|c|c|c|c|c|}
\hline \multirow[b]{2}{*}{ Enzyme } & \multicolumn{2}{|c|}{1} & \multicolumn{2}{|c|}{$\|$} \\
\hline & Activity & Controls & Activity & Controls \\
\hline NADH dehydrogenase & 31.4 & $12-40$ & 0.28 & $0.17-0.56$ \\
\hline Citrate synthase & 113.0 & $45-105$ & & \\
\hline
\end{tabular}

$I=$ activity expressed as a function of total cellular protein ( $\mu \mathrm{mol} / \mathrm{min} / \mathrm{g}$ protein).

II = activity when expressed on the reference enzyme citrate synthase, a mitochondrial matrix marker $(\mu \mathrm{mol} / \mathrm{min} / \mu \mathrm{mol} / \mathrm{min} \mathrm{citrate}$ synthase $)$.

$\mathrm{NADH}=$ reduced nicotinamide-adenine dinucleotide. 
$\mathrm{mmol} / \mathrm{L}$ and pyruvate $0.114 \mathrm{mmol} / \mathrm{L}$ with a lactate/pyruvate ratio of 24 (nor$\mathrm{mal}<20$ ). Biogenic amines in the cerebrospinal fluid were normal. Serum creatine kinase was repeatedly elevated up to $635 \mathrm{U} / \mathrm{L}$ (normal $<107 \mathrm{U} / \mathrm{L}$ ). The optic fundi were normal, as was the electroencephalogram. Signs of mild concentric hypertrophic cardiomyopathy were present on echocardiography, and the electrocardiogram was normal. Visual, auditory, and median nerve somatosensory evoked potentials fell within normal ranges, whereas tibial nerve somatosensory evoked potential was pathologic. Motor and sensory nerve conduction velocities were slow, indicating mild demyelinating peripheral neuropathy. Subsequent cranial MRI performed at the age of 19 years showed changes described as previously, revealing abnormal signal density in the structures of basal ganglia on MRI fluid-attenuated inversion-recovery sequences (Figure 3), whereas no abnormal signal could be seen in the cerebral peduncles (Figure 4). A muscle biopsy revealed mild fiber atrophy, with necrotic fibers undergoing phagocytosis and no raggedred fibers. The measured catalytic activity of cytochrome $c$ oxidase in skeletal muscle homogenates demonstrated a partial cytochrome $c$ oxidase deficiency (Table 1). Leukocyte mitochondrial DNA demonstrated no point mutations at nucleotides 3243,8993 , and 8344 , and no mitochondrial deletions/duplications were found by both polymerase chain reaction and Southern blotting. SURF-1 analysis was performed according to Tiranti. ${ }^{14}$ Sequence analysis of exons 1 to 8 showed no abnormalities.

The boy is receiving levodopa therapy $(2 \times 100 \mathrm{mg})$ and high doses of thiamine ( $3 \times 100 \mathrm{mg}$ ) and lipoic acid ( $30 \mathrm{mg} / \mathrm{kg} /$ day) and was placed on a ketogenic diet for several years subsequently. Recently, a creatine monohydrate was introduced ( $2 \times 2 \mathrm{~g} /$ day, 6 days per week) and his condition remains stabilized, even showing improvement in his muscle performance and strength but less pronounced extrapyramidal manifestations.

\section{Discussion}

Cytochrome $c$ oxidase deficiency is the most common biochemical defect associated with Leigh disease. ${ }^{7}$ Since Willems et al reported the first case in 1977 , numerous cases with cytochrome $c$ oxidase-associated Leigh disease have been reported, with a similar clinical course ${ }^{6,8}$ Most patients have normal neurologic development during the first 8 to 12 months, with common somatic complaints including recurrent vomiting, chronic diarrhea, anorexia, and decelerating body and head growth. The second stage evolves during late infancy and early childhood, when motor regression becomes evident. Pyramidal, extrapyramidal, and cerebellar signs, altered breathing patterns, and ophthalmoplegia appear, and sudden clinical deterioration occurs during intercurrent infections or metabolic stress. In the last stage of varying duration, extreme hypotonia, swallowing difficulties, and undernutrition dominate. Systemic cytochrome $c$ oxidase deficiency presenting as Leigh disease is inherited as an autosomal-recessive trait; however, the underlying molecular defect remains unknown. Cytochrome $c$ oxidase activity in these patients is reduced in all tissues of the body, often to very low residual levels, with little or no tissue specificity in the severity of the defect. ${ }^{17,18}$ Cytochrome $c$ oxidase multisubunit complex is the terminal electron acceptor of the mitochondrial respiratory chain composed of 13 subunits, 3 of which are encoded by the mitochondrial genome and form the catalytic core of the enzyme. The remaining 10 subunits are encoded by the nuclear genome. Somatic cell genetic studies have demonstrated that the majority of patients with the cytochrome $c$ oxidase-deficient Leigh disease belong to a single genetic complementation group. ${ }^{19}$ DNA sequence analysis of complementary DNA for all 13 structural subunits of the cytochrome $c$ oxidase complex has not revealed any pathogenic mutations, suggesting that a failure to assemble an active enzyme complex is the basis of the lack of enzyme activity in most cases. ${ }^{18,20}$ Recently, Zhu et al mapped the gene defect in Leigh disease to chromosome 9q34 by complementation of the respiratory chain enzyme deficiency in patient fibroblasts using microcellmediated chromosome transfer. ${ }^{21}$ DNA sequence analysis of a candidate gene (SURF-1) in the region revealed several different pathogenic mutations, all of which predicted a truncated protein. Their data indicated that $S U R F-1$ encodes a putative assembly or maintenance factor that, in humans, appears to be specific for the cytochrome $c$ oxidase complex. ${ }^{21}$

We present a boy with partial cytochrome $c$ oxidase deficiency who had normal developmental skills in the first $2 \frac{1 / 2}{2}$ years of life. His condition worsened after a minor respiratory infection, when he developed signs of cerebellar dysfunction with gait ataxia and tremor. CT of the brain performed shortly after the onset of the symptoms revealed cerebellar atrophy. His further clinical course was marked by development of generalized dystonia; choreoathetosis of the arms, hands, legs, and feet; transient dysphagia; ptosis and convergent strabismus; progressive dysarthria; nystagmus with signs of demyelinating peripheral neuropathy; and pyramidal dysfunction resulting from lesions in the basal ganglia, cerebellum, and brain stem as demonstrated on subsequent CT and MRI brain scans. Biochemical investigation displayed mild to moderate elevation of serum lactate and cerebrospinal fluid lactate and pyruvate levels. Extrapyramidal signs, such as generalized dystonia, tremor, chorea, and athetosis, were the first symptoms in our patient. These are typical symptoms in Leigh disease as a consequence of basal ganglia involvement. ${ }^{15,22}$ The enzyme defect most frequently associated with dystonia is cytochrome $c$ oxidase deficiency of variable degree; this was also present in our patient. As is the case in half of all patients with Leigh disease caused by cytochrome $c$ oxidase deficiency, ${ }^{11.21}$ no abnormalities in the $S U R F-1$ gene were found in the patient described here. This does not exclude mutations within the introns or at the splice sites of the $S U R F-1$ gene or in other genes involved in the biogenesis and regulation of cytochrome $c$ oxidase.

After diagnostic procedures, a therapeutic attempt with levodopa was started. A fixed combination of levodopa and carbidopa was introduced, and after a short period of time, the clinical condition of our patient improved significantly. Dystonia and choreoathetosis of the lower extremities were less pronounced, and the patient's walking pattern ameliorated. We found that dystonia only involving the facial musculature was unresponsive to this therapy. Recently, an oral supplementation of creatine monohydrate was added to the therapy of our patient according to Tarnopolsky et al, and the beneficial clinical effects have already been reported. The patient's condition has been stable for the past 4 years, and no adverse reactions have occurred.

Melita Čačić, MD, $\mathrm{PhD}$
Department of Pediatrics
Children's Hospital Zagreb
Ekkehard Wilichowski, MD, $\mathrm{PhD}$
Department of Neuropediatrics
Georg-August University Göttingen
Göttingen, Germany
Vlatka Mejaški-Bošnjak, MD, PhD
Department of Pediatrics
Children's Hospital Zagreb

Melita Čačić, MD, PhD Children's Hospital Zagreb

Ekkehard Wilichowski, MD, PhD

Department of Neuropediatrics

Georg-August University Göttingen

Göttingen, Germany

Department of Pediatrics

Children's Hospital Zagreb 


\author{
Ksenija Fumić, PhD \\ Department of Biochemistry \\ Clinical Hospital Center Zagreb \\ Lucija Lujić, MD \\ Branka Marušić Della Marina, MD, MSc \\ Department of Pediatrics \\ Children's Hospital Zagreb \\ Zagreb University Medical School \\ Zagreb, Croatia \\ Folker Hanefeld, MD, PhD \\ Department of Neuropediatrics \\ Georg-August University Göttingen \\ Göttingen, Germany
}

Received April 27, 2000. Received revised Dec 7, 2000. Accepted for publication Dec 8, 2000.

This work was presented in part at the 8th International Child Neurology Congress in Ljubljana, Slovenia, 1998.

Address correspondence to Dr Vlatka Mejaški-Bošnjak, Children's Hospital Zagreb, Klaiceva 16, 10000 Zagreb, Croatia. Tel: 385-1-4600-137; fax: 385-14600-160; e-mail: vbosnjak@kdb.hr.

\section{References}

1. Leigh $\mathrm{D}$ : Subacute necrotizing encephalomyelopathy in an infant. $J$ Neurol Neurosurg Psychiatry 1951;14:216-221.

2. Van Erven PMM, Cillessen JPM, Eekhoff EMW, et al: Leigh syndrome, a mitochondrial encephalom(myo)pathy. A review of the literature. Clin Neurol Neurosurg 1987;89:217-230.

3. DiMauro S, De Vivo DC: Genetic heterogeneity in Leigh syndrome. Ann Neurol 1996;40:5-7.

4. De Vivo DC, Haymond MW, Obert KA, et al: Defective activation of the pyruvate dehydrogenase complex in subacute necrotizing encephalomyelopathy (Leigh disease). Ann Neurol 1979;6:483-494.

5. Korenke G-C, Bentlage HACM, Ruitenbeek W, et al: Isolated and combined deficiencies of NADH dehydrogenase (complex I) in muscle tissue of children with mitochondrial myopathies. Eur J Pediatr 1990;150:104-108.

6. Willems JL, Monnens L, Trijbels J, et al: Leigh's encephalomyelopathy in a patient with cytochrome $c$ oxidase deficiency in muscle tissue. Pediatrics 1977;60:850-857.

7. DiMauro S, Servidei S, Zeviani M, et al: Cytochrome $c$ oxidase deficiency in Leigh syndrome. Ann Neurol 1987;22:498-506.

8. Van Coster R, Lombes A, De Vivo DC, et al: Cytochrome $c$ oxidase-associated Leigh syndrome: Phenotypic features and pathogenetic speculations. $J$ Neurol Sci 1991;104:97-111.

9. Adams PL, Lightowlers RN, Turnbull DM: Molecular analysis of cytochrome $c$ oxidase deficiency in Leigh's syndrome. Ann Neurol 1997;41:268-270.

10. Baumgartner ER, Sourmala TM, Wick H, et al: Biotinidase deficiency: A cause of subacute necrotizing encephalomyelopathy (Leigh syndrome). Report of a case with lethal outcome. Pediatr Res 1989;26:260-266.

11. Shoffner JM, Fernhoff MD, Krawiecki NS, et al: Subacute necrotizing encephalopathy: Oxidative phosphorylation defects and the ATPase 6 point mutation. Neurology 1992;42:2168-2174

12. Chalmers RM, Lamont PJ, Nelson I, et al: A mitochondrial DNA tRNA(Val) point mutation associated with adult-onset Leigh syndrome. Neurology 1997;49:589-592.

13. Bourgeron T, Rustin P, Chretien D, et al: Mutation of a nuclear succinate dehydrogenase gene results in mitochondrial respiratory chain deficiency. Nat Genet 1995;11:144-149.

14. Tiranti V, Hoertnagel $\mathrm{K}$, Carrozzo $\mathrm{R}$, et al: Mutations of $S U R F-1$ in Leigh disease associated with cytochrome $c$ oxidase deficiency. Am J Hum Genet 1998;63:1609-1621

15. Rahman S, Blok RB, Dahl H-HM: Leigh syndrome: Clinical features and biochemical and DNA abnormalities. Ann Neurol 1996;39:343-351.
16. Macaya A, Munell F, Burke RE, De Vivo DC: Disorders of movement in Leigh syndrome. Neuropediatrics 1993;24:60-67.

17. Miranda AF, Ishii S, DiMauro S, Shay JW: Cytochrome $c$ oxidase deficiency in Leigh's syndrome: Genetic evidence for a nuclear DNAencoded mutation. Neurology 1989;39:697-702.

18. Lombes A, Nakase H, Tritschler H-J, et al: Biochemical and molecular analysis of cytochrome $c$ oxidase deficiency in Leigh's syndrome. Neurology 1991;41:491-498.

19. Brown RM, Brown GK: Complementation analysis of systemic cytochrome oxidase deficiency presenting as Leigh syndrome. $J$ Inherit Metab Dis 1996;19:752-760.

20. Hayasaka K, Brown GK, Danks DM, et al: Cytochrome c oxidase deficiency in subacute necrotizing encephalopathy (Leigh syndrome). $J$ Inherit Metab Dis 1989;12:247-256.

21. Zhu Z, Yao J, Johns T, et al: $S U R F 1$, encoding a factor involved in the biogenesis of cytochrome $c$ oxidase, is mutated in Leigh syndrome. Nat Genet 1998;20:337-343.

22. Munnich A, Rötig A, Chretien D, et al: Clinical presentation of mitochondrial disorders in childhood. J Inherit Metab Dis 1996;19:521-527.

23. Tarnopolsky MA, Roy BD, Donald JR: A randomized, controlled trial of creatine monohydrate in patients wtih mitochondrial cytopathies. Muscle Nerve 1997;20:1502-1509.

\section{Neonate With Spinal Hypoplasia on T12 and a Localized Vertebral Malformation on $\mathbf{L 4}$}

\section{ABSTRACT}

We report a case of a neonate with sectional narrowing of the spinal cord on the level of $\mathrm{T} 12$ to $\mathrm{L} 2$ and a deformed vertebral body on a different level, L4. In previously described cases of sectional spinal dysgenesis, the vertebral and spinal cord malformations are usually found on the same level. Our case may represent a new variant of spinal dysgenesis. (J Child Neurol 2001;16:619-621).

Segmental spinal dysgenesis is a rare condition first defined by Scott et al in $1988^{1}$ and characterized by focal agenesis or dysgenesis of the thoracolumbar or lumbar spine. Usually, there is a significant narrowing of the thecal sac and an absence of adjacent nerve roots at the level of the lesion. The clinical picture usually parallels the severity of the malformation and its sectional level along the longitudinal embryonic axis.

We describe an unusual case of spinal dysgenesis with a localized vertebral anomaly and, on a different spinal level, a hypoplasia of the spinal cord

\section{Case Report}

After 38 weeks' gestation, a boy weighing $3630 \mathrm{~g}$ (50th percentile) (Figure 1) was born to a 25 -year-old nondiabetic mother by cesarean section because of maternal preeclampsia. There was no history of drug intake during the first weeks of pregnancy.

Cardiopulmonary adaptation of the infant was unremarkable. The clinical examination revealed lower extremity palsy, clubfeet, nonfixed contractions of the hips and knees, and a head circumference of $38 \mathrm{~cm}$ (>90th percentile). Reduced muscular activity with rare spontaneous movements of the lower limbs, sensory loss below the area supplied by L3, and reduced muscle tendon reflexes were noted. The rectus abdominus muscular activity and anal sphincter tone were within normal limits. No abnor- 